\title{
Energy Efficient Optimization of a Sleep Mode Strategy in Heterogeneous Cellular Networks
}

\author{
Marco Dolfi*, Simone Morosi*, Cicek Cavdar ${ }^{\dagger}$ and Enrico Del Re* \\ *Department of Information Engineering \\ University of Florence, Florence, Italy \\ Email: marco.dolfi@unifi.it, simone.morosi@unifi.it, enrico.delre@unifi.it \\ †Communication Systems Department, \\ KTH Royal Univesity of Technology, Kista, Sweden \\ Email: cavdar@kth.se
}

\begin{abstract}
As base stations (BS) are responsible for the large amount of energy consumed in cellular networks, energy efficient BS sleep mode techniques have the potential to save a significant amount of energy. However, assuming that BSs are able to alternate between sleeping and active states as frequently as possible may have a negative impact on network reliability, shortening BS lifetime. In this paper we propose a multiobjective optimization framework aimed at minimizing the power consumption and number of BS sleep mode switchings in heterogeneous cellular networks (HetNet), by jointly considering Quality of Service (QoS) requirements. We focus on the HetNet scenario in which macro and micro cells coexist. The Mixed Integer Quadratic Programming (MIQP) optimization technique is used to minimize the power consumption together with the number of BS sleep mode operations of both macro and micro cells. The tradeoff between power consumption, sleep mode switchings and performance of the network is shown for different energy saving solutions. Results show that the proposed optimization can guarantee QoS target throughput for users and significant reduction of $50 \%$ for macro and $73 \%$ for micro BS respectively daily number of switchings, while still achieving $8 \%$ savings in terms of daily energy consumption.
\end{abstract}

\section{INTRODUCTION}

Mobile operators need to seek "green" solutions in order to reduce operating expense (OPEX) and contain the energy consumption of future mobile networks, which will feature very high technical goals, such as higher user data rates, improved coverage with uniform user experience and reduced end-to-end latency. Recent studies have explored adaptive radio resource management (RRM) solutions to save energy and improve network utilization efficiency. When the spatial traffic distribution is non-uniform and time-varying, dynamic coverage management may be introduced to exploit traffic variations. Dynamic switch on/off of coverage overlaid cells in low traffic is an example. By adopting this solution the BS activity could be adapted to the traffic demand avoiding the waste of energy due to the peak dimensioning [1], [2]. The combination of different access points, traffic loads and radio access technologies, makes the network highly heterogeneous. Hence, the same deployment strategy cannot be used everywhere and the same RRM solution cannot be used throughout the day with very different network conditions. In [3] analytical models are developed to identify optimal fixed BS switch-off times as a function of the daily traffic pattern. Dynamic sleep mode schemes generally require more switching operations as compared to fixed schemes, especially with highly variable traffic patterns. Therefore, a fundamental trade-off to be considered is between more energy saving in sleep mode and the cost of switching operations, which includes extra power for monitoring and switching, overhead, delay constraints, and impact on the operational lifetime of BSs. One common problem with current research in this area is that most work implicitly assume that BSs are able to alternate between sleeping and active mode as frequently as possible [4]. It is worth mentioning that BS sleeping might negatively impact QoS requirements of the system because of decreasing capacity, unless specific remedial actions are adopted concurrently [5]. In general, there is no available closed form expression to show the direct relation between transmit power and QoS and user experience measures, such as service latency or user perceived throughput. This aspect calls for design and optimization frameworks that handle multiple objectives and support the selection of the best attainable operating point [6]-[8].

In this paper an energy efficient and adaptive cellular network configuration strategy with QoS requirements is investigated. The solution is based on the use of traffic forecast in order to allow the base stations to know the traffic behavior in their coverage area. A given service rate is guaranteed to mobile terminals and the cost of rearranging the network when traffic demand changes is taken into account by optimizing the actual number of BS switchings, defined as the number of active/inactive state transitions in a twenty-four hour period. As highlighted in [9], the forecast approach requires a lower number of switch on/off operations with respect to the procedure which is based on instantaneous traffic measurements; as a result, the control traffic and handover operations are also reduced. This work extends the results presented in [10] by considering a multiobjective optimization framework, designed to inspect the network sleep mode operation cost over a daily pattern of traffic demands. With the introduction of small cell overlays, the macro cell network becomes overprovisioned due to the offload of traffic by means of small cells. One strategy for the network operator is to keep the 


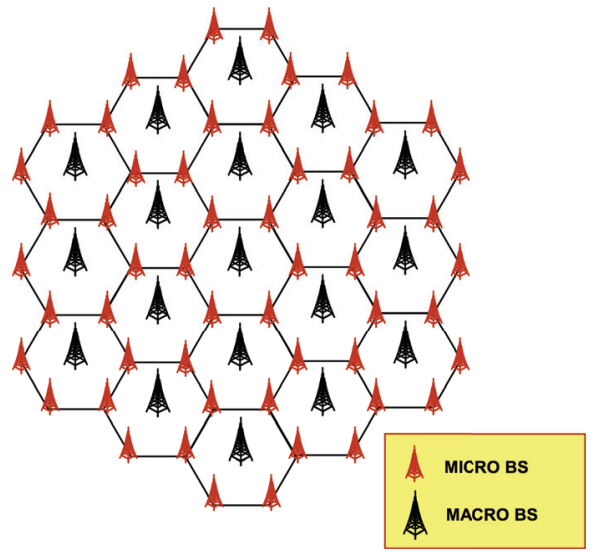

Fig. 1. Considered het-net scenario

existing macro cell BSs as they are, until the natural growth in user demand catches up with the spare capacity. This approach does not offer the most efficient energy saving solution since it may take a long time for growth in user demand to increase sufficiently. Alternatively, the network operator can re-optimize the existing macro cell network in response to small cell deployment. Performing this optimization will make the overall network more energy efficient and reduce network OPEX over the long term. The small cells can improve network performance and service quality by offloading from the large macro cells, but a negative effect is the increased interference on the downlink experienced by the user. These questions call for handling the network deployment in a more efficient way, by closely reexamining its requirements. The optimization problem formulation assumes weighting factors between the conflicting objectives of reducing the power consumption while narrowing down sleep mode operations. Both macro and micro cells subsets are jointly considered in the sleep mode scheme of the optimization process, allowing to avoid overlapping coverage. The paper is organized as follows: Section II describes the system model and the considered scenario; in Section III the optimization problem is formulated; Section IV describes the adopted power saving strategies and Section V discusses about the simulation results. Finally, Section VI concludes the work.

\section{SYSTEM MODEL}

A LTE-based network of two different layers of hexagonal cells is considered, as shown in Figure 1. The first layer is composed of a set of 19 macro cells, while the second layer is formed by 54 micro cells surrounding the first macro cell layer. Both macro and micro base stations are equipped with omnidirectional antennas. According to [11], for each cell the following power model is considered:

$$
P_{c}= \begin{cases}a \cdot P_{t x}+P_{0}, & \text { if } \mathrm{BS} \text { is } \mathrm{ON} \\ P_{\text {sleep }}, & \text { if } \mathrm{BS} \text { is } \mathrm{OFF}\end{cases}
$$

In particular the value of $a, P_{0}$ and $P_{\text {sleep }}$ are related respectively to the variable power consumption, the fixed power consumption of the active base station and the fixed
TABLE I

POWER CONSUMPTION MODEL PARAMETERS

\begin{tabular}{lccc}
\hline \hline Bs type & $\mathbf{a}$ & $\mathbf{P}_{\mathbf{0}}$ & $\mathbf{P}_{\text {sleep }}$ \\
Macro BS & 4.7 & $130 \mathrm{~W}$ & $75 \mathrm{~W}$ \\
Micro BS & 2.6 & $56 \mathrm{~W}$ & $39 \mathrm{~W}$ \\
\hline \hline
\end{tabular}

TABLE II

GIVEN DATA FOR THE CONSIDERED PROBLEM

\begin{tabular}{ll}
\hline Parameter & Value \\
\hline$N$ & number of deployed base stations \\
$M$ & number of users \\
$\mathcal{B}=\left\{B S_{1}, \ldots, B S_{N}\right\}$ & set of $N$ deployed base stations \\
$\mathcal{U}=\left\{U E_{1}, \ldots, U E_{M}\right\}$ & set of $M$ users which have to be served \\
$P_{M I N j}$ & sensitivity of UE $j$ \\
$P_{M A X}$ & maximum allowed BS transmission power \\
$R_{t}$ & datarate target for each UE \\
$N_{p}$ & number of available PRBs at BS \\
$W$ & total available bandwidth at BS \\
$W_{p}$ & bandwidth of a single PRB \\
$\sigma_{i j}$ & channel gain between BS $i$ and UE $j$ \\
\hline \hline
\end{tabular}

power consumption of the base station on sleep mode. These parameters are set as described in Table I for a typical LTE system.

Regarding the traffic generation, each user in the interested area requests a constant bitrate data stream: if the target data rate value is reached, the quality of the link is assumed to be acceptable. Each user can be served by macro and micro base stations, but can be connected to only one base station at a time. In [11] the variations of traffic data during the day are modeled with a daily pattern related to the percentage of active users during the day and the global number of subscribers in a certain area. In this work the average number of simultaneous users at busy hour has been fixed and then calculated following this pattern for the rest of the day. The number of simultaneous users is assumed to follow a Poisson distribution with a different mean at each hour. The users are uniformly distributed in the considered area. In order to exploit the het-net scenario in terms of effective macro/micro cell sleep mode transitions, a suitable traffic forecast technique is considered [2].

\section{Problem Formulation}

Given the system described in Sec. II and the data reported in Table II, the goal of the problem is to minimize the global power consumption $P_{c}$ while controlling the number of BS sleep mode switchings $S$ during daily traffic variations. Let $\mathcal{B}=\left\{B S_{1}, \ldots, B S_{N}\right\}$ and $\mathcal{U}=\left\{U E_{1}, \ldots, U E_{M}\right\}$ be respectively the set of $N$ deployed base stations and the set of $M$ users which have to be served. The binary variable $x$ represents the association between BSs and UEs, as in the following:

$$
x_{i j}=\left\{\begin{array}{ll}
1 & \text { if UE } j \text { is served by BS } i \\
0 & \text { otherwise }
\end{array} \quad i \in \mathcal{B}, j \in \mathcal{U}\right.
$$

Assuming $\pi_{i j}$ as the power assigned for transmission between BS $i$ and UE $j$ and $w_{i j}$ as the bandwidth assigned by $\mathrm{BS} i$ to $\mathrm{UE} j$, the data rate achieved by $\mathrm{UE} j$ is:

$$
\rho_{j}=\sum_{i \in \mathcal{B}} x_{i j} w_{i j} \log _{2}\left(1+\gamma_{i j}\right)
$$


where $\gamma_{i j}$ is the SINR between BS $i$ and UE $j$. The transmission power of each BS $i$ can be calculated as $P_{i}=$ $\sum_{j \in \mathcal{U}} \pi_{i j} x_{i j}$. Therefore, the SINR $\gamma_{i j}$ is

$$
\gamma_{i j}=\frac{\pi_{i j} \sigma_{i j} x_{i j}}{\frac{w_{i j}}{W}\left(\sum_{k=1}^{N} P_{k} \sigma_{k j} \zeta_{k}\left(1-x_{k j}\right)+W N_{0}\right)}
$$

where $\sigma_{i j}$ is the channel gain between $\operatorname{BS} i$ and UE $j, W$ is the total available bandwidth at $\mathrm{BS}$ and $N_{0}$ is the noise spectral density. The activity status of each BS is modeled by the binary variable $\zeta$ :

$$
\zeta_{i}=\left\{\begin{array}{ll}
1 & \text { if BS } i \text { is active } \\
0 & \text { if BS } i \text { is in SLEEP mode }
\end{array} \quad i \in \mathcal{B}\right.
$$

Let $\mathcal{T}=\left\{t_{1}, \ldots, t_{L}\right\}$ be the set of $L$ traffic demand forecasts during the day in terms of UEs to be served. At every time $t \in \mathcal{T}$ the two objective functions are then calculated as:

$$
\begin{aligned}
& P_{c}^{(t)}=\sum_{i=1}^{N}\left[\left(a \sum_{j=1}^{M} \pi_{i j} x_{i j}+P_{0}\right) \zeta_{i}+\left(1-\zeta_{i}\right) P_{\text {sleep }}\right] \\
& S^{(t)}=\sum_{i \in \mathcal{B}}\left[\zeta_{i}^{(t)}\left(1-\zeta_{i}^{(t-1)}\right)+\zeta_{i}^{(t-1)}\left(1-\zeta_{i}^{(t)}\right)\right]
\end{aligned}
$$

where parameters $a, P_{0}$ and $P_{\text {sleep }}$ are the slope of the dynamic consumption, the fixed consumption and the sleep mode consumption, respectively [12]. Considering the activity status transitions of each macro and micro BS in response to the changing traffic demand, eq. (6b) keeps track of the number of sleep mode operations triggered by the energy efficiency policies. We assume two weighting factors, $\lambda_{M}$ and $\lambda_{m}$, in the energy efficiency optimization process, in order to control the number of macro and micro BS subsets' sleep mode operations, $S_{M}$ and $S_{m}$, respectively. The optimization problem is formulated in (7a)-(7h).

$$
\begin{array}{ll}
\min _{\pi, x, \zeta} \quad & \left(P_{c}+\lambda_{M} S_{M}+\lambda_{m} S_{m}\right) \\
\text { s.t. } & \sum_{i=1}^{N} x_{i j}=1, \quad \forall j \in \mathcal{U}, \\
& \sum_{i=1}^{N} \sum_{j=1}^{M} x_{i j}=M, \quad \forall j \in \mathcal{U}, \\
& \sum_{j=1}^{M} x_{i j} \leq N_{P R B}, \quad \forall i \in \mathcal{B}, \\
& c_{i j} \leq \frac{\pi_{i j} \cdot \sigma_{i j}}{P_{M I N j}}, \quad \forall i \in \mathcal{B} \quad \forall j \in \mathcal{U}, \\
& c_{i j}-x_{i j} \geq 0, \quad \forall i \in \mathcal{B} \quad \forall j \in \mathcal{U}, \\
& \zeta_{i} \leq x_{i j}, \quad \forall j \in \mathcal{U} \quad \forall i \in \mathcal{B}, \\
& \sum_{j=1}^{M} \pi_{i j} \leq P_{M A X}, \quad \forall i \in \mathcal{B} .
\end{array}
$$

Constraints (7b) and (7c) determine the coverage and the singular association for each UE. Constraint (7d) sets the BS capacity limit, in terms of bandwidth elements $N_{P R B}$. Constraint (7e) is fundamental for assuring the QoS: the binary variable $c_{i j}$ equals to 0 if $\pi_{i j} \sigma_{i j} \leq P_{M I N j}$; hence, for a given UE, constraint (7e) will define the set of potential BSs
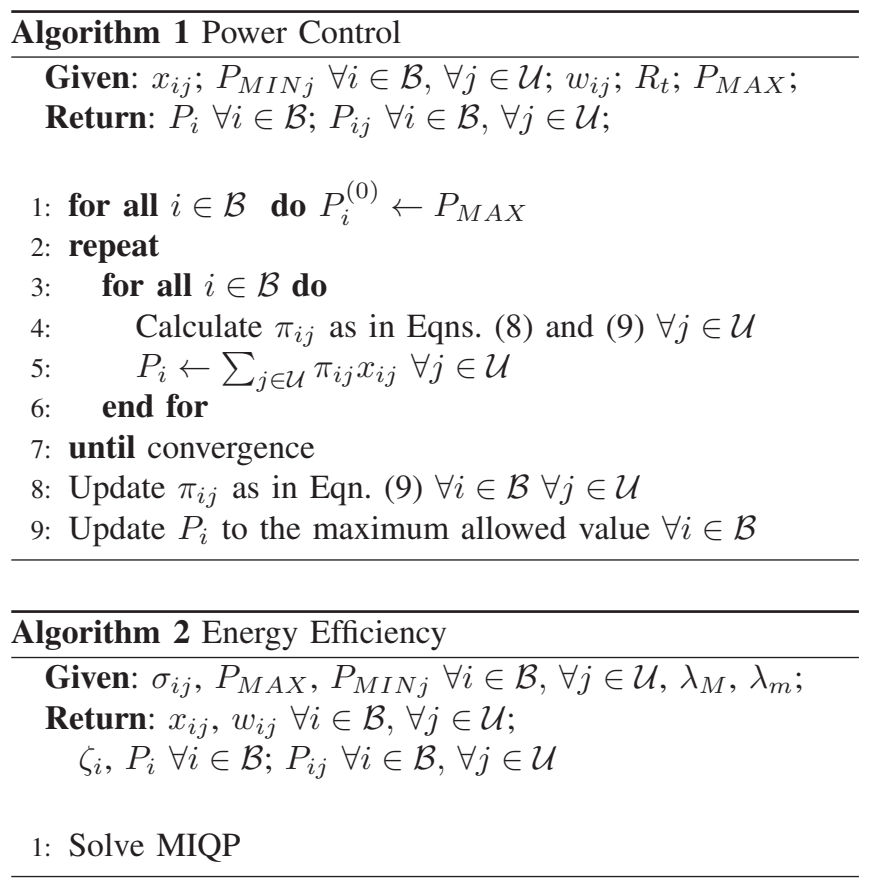

that can provide the minimum received power, $P_{M I N j}$. Then, introducing constraint (7f), only one of the BSs in this set is selected. The activity status of a base station is linked to the user associations by constraint $(7 \mathrm{~g})$. Finally, constraint (7h) sets the limit on the maximum BS transmission power.

\section{NETWORK OPTIMIZATION SOLUTIONS}

\section{A. Power control}

Power control is a well known solution that is able to decrease the global energy consumption by reducing the inter cell interference. As shown in Alg. 1, in this work the considered power control algorithm is based on the UE-BS association and the bandwidth assignment for each UE. The target QoS data rate for each UE is guaranteed by the iterative process, which provides the optimum BS transmission power. The proof of convergence can be found in [13]. At each iteration $n$ the power transmitted by a BS to a certain UE is calculated as

$$
\pi_{i j}^{(n)}=\frac{w_{i j} 2^{\frac{R_{t}}{w_{i j}}}}{W \sigma_{i j}}\left(\sum_{k \in \mathcal{B}} P_{k}^{(n-1)}\left(1-x_{k j}\right) \sigma_{k j}+W N_{0}\right)
$$

where $R_{t}$ is the target data rate, i.e. the QoS requirement for each UE. The initial condition is $\sum_{j} \pi_{i j}^{(0)}=P_{M A X}$ for all $i \in \mathcal{B}$. The received power for each UE $j$ must be greater than the sensitivity $P_{M I N j}$ : if this is not the case the power which is transmitted by a BS to a certain UE is adjusted by the following equation:

$$
\pi_{i j}=\max \left(\frac{P_{M I N j}}{\sigma_{i j}} ; \pi_{i j}\right)
$$




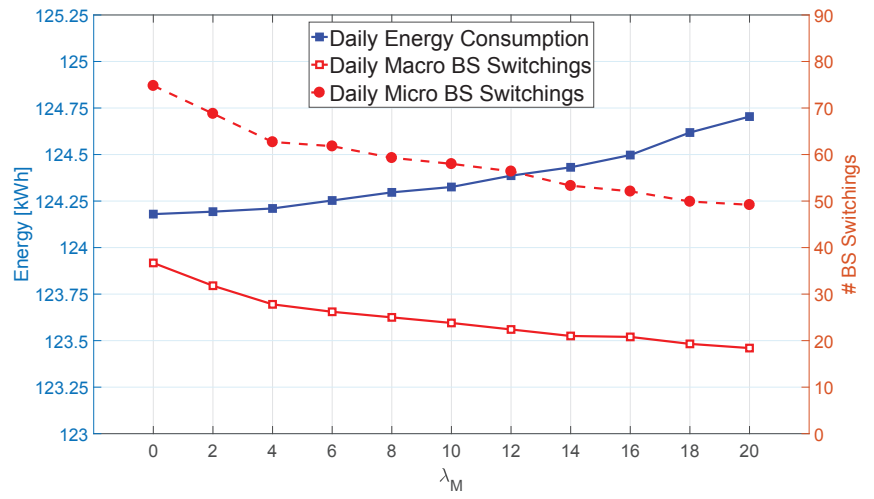

(a)



(b)

Fig. 2. Energy consumption vs BS switchings in the optimization process: (a) Macro BS subset sleep mode operations weighting factor $\lambda_{M}$; (b) Micro BS subset sleep mode operations weighting factor $\lambda_{m}$

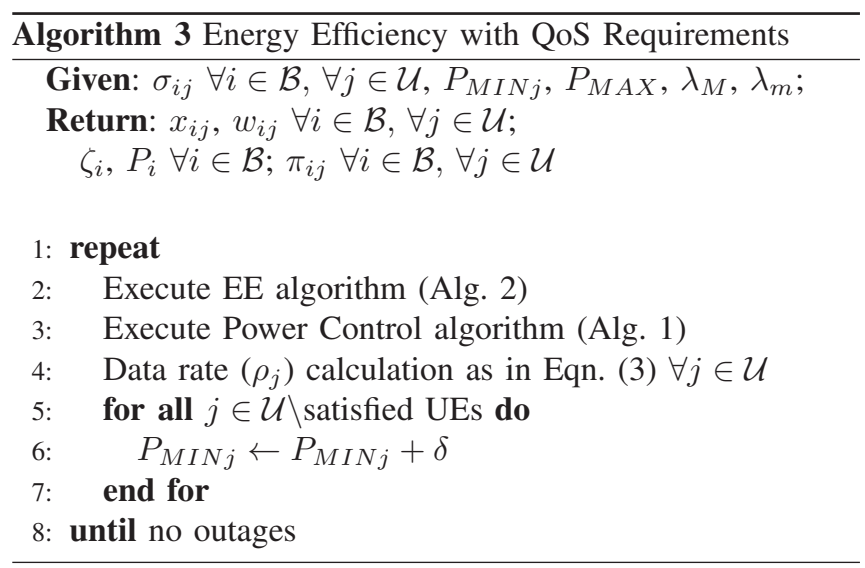

\section{B. Het-Net EE optimization}

The fundamental approach of the optimization problem is to recognize the existence of multiple objectives, such as guaranteed rate for all the users, network power consumption, number of BS sleep mode operations and number of simultaneously active BSs, both of macro and micro cell subsets. In order to minimize the power consumption in the cellular network, a first optimization strategy is proposed. The iterative process solves the problem for three variables: association between $\mathrm{BS}$ and UE, bandwidth assignment and power allocation. In order to limit the cost of sleep mode operations, the number of power state transitions of BSs is taken into account in the optimization process. The deactivation and power reduction of the BSs are allowed only if the target QoS requirement is satisfied for each served UE. A MIQP solver has been adopted to perform the optimization procedure that refers to the problem formulated in (7a)-(7h). The MIQP model is solved by IBM ILOG CPLEX ${ }^{\circledR}$ solver [14]. Since the model cannot manage directly the QoS for each UE because of its non-linearity, two approaches are proposed in order to avoid any outage: (i) Power consumption minimization assuming an interference controlled scenario $(E E)$; (ii) Iterative power consumption and BS sleep mode operation minimization to guarantee QoS ( $E E$ $Q o s)$. The $E E$ scenario assumes a perfect inter cell interference cancellation (ICIC) solution. If the interference cannot be neglected, the $E E$ algorithm cannot guarantee the required QoS and some outages could arise. Therefore, this energy saving solution represents an optimum lower bound in terms of global power consumption. In order to avoid the datarate outages and reduce the impact of sleep mode operations the $E E$ Qos strategy is introduced in Alg. 3. It combines the optimum power control and the $E E$ solutions in an iterative method. In particular the $E E$ algorithm obtains the optimum set of active BSs and the optimum BS-UE association, while the feasibility of the solution is controlled by the Power Control procedure as shown in Alg. 3. If some data rate outages occur, the power of the outage users is increased by a $\delta$ value in order to look for new active BS subsets and a better association. It is important to emphasize that although the $E E$ method provides more energy efficient sleep mode solutions, it incurs control signaling over the network to wake up cells. As an example, a single wake-up control packet could be used to trigger the activation/deactivation of a cell BS. The number of BS sleep mode operations related to the adoption of the energy saving strategies throughout the daily pattern of traffic demand forecasts is considered in the optimization process by computing a discrete set of $\lambda_{M}$ and $\lambda_{m}$ sample points. $\lambda_{M}$ and $\lambda_{m}$ introduce a weighting condition between the objective functions calculated in (6a), (6b) and (7a). As $\lambda_{M}$ and $\lambda_{m}$ increase, also the priority of reducing network operation and maintenance costs increases, for macro and micro BS subsets respectively.

\section{Simulation RESUlts}

The proposed energy saving solution, namely the EE Qos strategy is compared to upper and lower bound solutions. As for the upper bound, the Closest BS Association is considered, adopting Alg. 1, based on power control with closest BS association to each UE. The BSs which are not serving any UE are put on sleep mode. As a lower bound, the optimum energy saving solution $E E$ is adopted. Simulation parameters are reported in Table III [15]. If a UE cannot achieve the data rate target, the minimum received power threshold, defined as $P_{M I N j}$ in constraint $(7 \mathrm{e})$, is increased by $\delta=1 \mathrm{~dB}$ in each iteration 
TABLE III

SIMULATION PARAMETERS

\begin{tabular}{ll}
\hline Parameter & Value \\
\hline Macro BS intersite distance & $1000 \mathrm{~m}$ \\
Micro BS intersite distance & $500 \mathrm{~m}$ \\
Path loss & $15.3+37.6 \log (d)$ (3GPP Typical Urban) \\
Shadow fading & std dev $8 \mathrm{~dB}$ \\
Indoor loss & $20 \mathrm{~dB}$ \\
Carrier frequency & $2 \mathrm{GHz}$ \\
Bandwidth & $10 \mathrm{MHz}(50 \mathrm{PRBs})$ \\
Max macro BS $P_{T X}$ & $20 \mathrm{~W}$ \\
Max micro BS $P_{T X}$ & $5 \mathrm{~W}$ \\
Noise PSD & $-174 \mathrm{dBm} / \mathrm{Hz}$ \\
UE sensitivity & $-90 \mathrm{dBm}$ \\
Target user datarate & $1 \mathrm{Mbps}$ \\
\hline \hline
\end{tabular}

of EE Qos, solving the MIQP model with this new setting. The maximum number of active UEs at peak hours is equal to 570. It corresponds to the maximum number of UEs that can be managed by the Closest BS Association strategy without any capacity outage, hence representing the maximum load of the cellular network. The results are obtained by statistical analysis of 50 simulation runs with a $95 \%$ confidence interval. In Fig.2(a) and 2(b) the results obtained by EE $Q o S$ with a set of increasing $\lambda_{M}$ and $\lambda_{m}$ values respectively are shown. As expected, the introduction of the weighting factors in the optimization process allows to heavily reduce the number of BS sleep mode operations during the day. With $\lambda_{M}=20$, the daily energy consumption sligthly rises $(0.5 \mathrm{kWh})$, while the number of macro BS switchings is reduced by half. Note that as $\lambda_{M}$ increases, also the number of switchings of micro BS subset decreases. This trend could be explained by the greater efficiency of a more stable network configuration and deployment: switching off some macro cells might bring more energy savings, but on the other hand the increasing number of active micro cells covering macro cells area might have a negative impact in terms of operational costs, especially in high traffic periods. This trend is no longer true if we consider the optimization process of reducing micro BS switching operations: particularly, Fig.2(b) shows that when $\lambda_{m}$ values are greater than 10 , the reduced number of micro cell sleep mode operations causes the negative effect of increasing the number of macro cell sleep mode activations. Given the high number of deployed micro cells, the optimization procedure is able to greatly reduce the number of micro BS switchings: with $\lambda_{m}=10$ micro BS sleep mode operations are reduced by three quarters at the cost of a daily energy consumption increase of $0.8 \mathrm{kWh}$. Based on these results, the values of $\lambda_{M}^{*}=20$ and $\lambda_{m}^{*}=10$ have been considered as optimal weighting factors for $E E Q O S$ solution. In Fig.3(a) the UE satisfaction rate is depicted for the maximum network load, starting from the $E E$ solution as first iteration value. It can be noted the impact of the number of active UEs on the number of iterations before the 0 outage objective is achieved. While the $E E$ strategy experiences outages, the EE $Q o S$ solution converges to 0 outage performance after a number of iterations that is related to the current network traffic load in terms of users. Each iteration corresponds to a solution in the search space for the MIQP model. The good behaviour of the iterative optimization procedure allows to obtain the target solution in

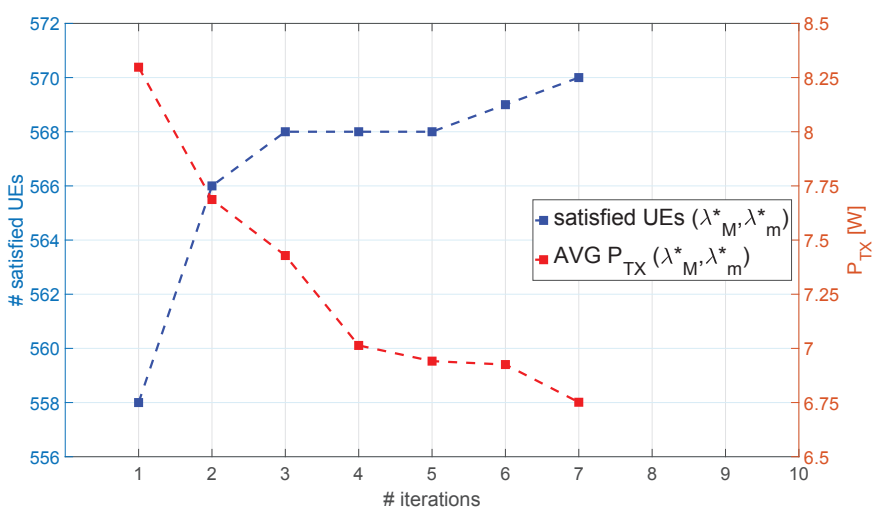

(a)

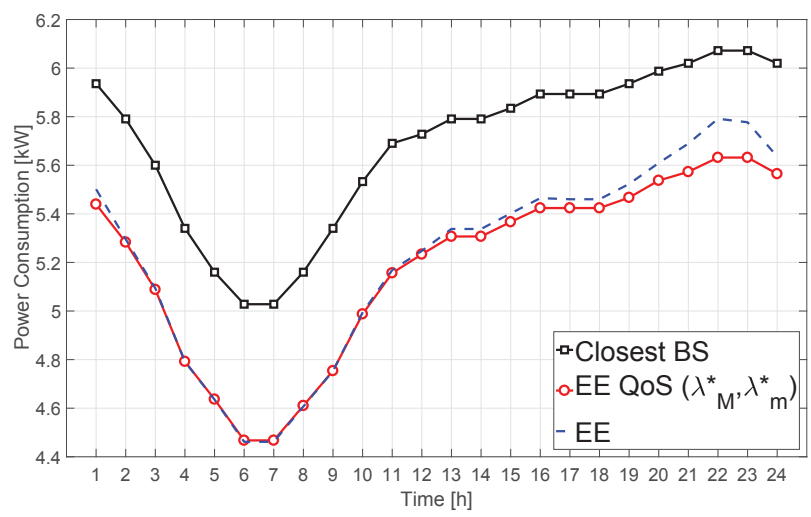

(b)

Fig. 3. Simulation results: (a) satisfied UEs and average transmission power per active BS vs number of iterations in EE Qos; (b) power consumption vs time of day for the implemented solutions

less than 10 iterations even in high traffic load case. The average transmission power vs the number of iterations is also depicted in Fig.3(a). Active UEs are satisfied when the mapping and received power allow to reach the QoS target. Among the feasible solutions, the one minimizing the global power consumption is chosen. In particular, even if the proper association is obtained by increasing the BS transmission powers, this trend becomes harmful for cell edge users because of the increasing interference level. In this case it is better to switch on other BSs, reducing the average transmission power together with the total power consumption and the interference. Fig.3(b) presents the results in terms of power consumption for EE $Q O S$ optimization strategy, with respect to the other solutions, i.e., Closest BS Association and EE. From the figure the behaviour of the proposed solution is evident: by introducing the optimal weights for macro and micro BS sleep mode operations in the optimization process, the number of active BSs ends up being heavily reduced, bringing high energy savings with respect to the Closest $B S$ Association. Because of the QoS requirements, the slope of the EE $Q O S$ solution is slightly higher in high traffic load periods with respect to the $E E$ performance: however it is interesting to see that the $E E Q O S$ results are very close to the optimum lower bound represented by the $E E$ strategy. In order to evaluate the overall throughput performance of the 


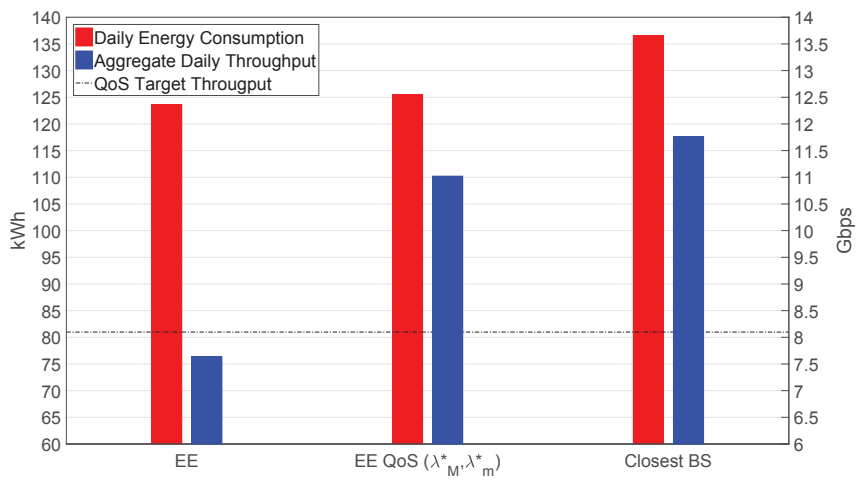

Fig. 4. Daily energy consumption and aggregate throughput

proposed solution, Fig. 4 shows the obtained results in terms of daily power consumption and aggregate daily throughput. While the EE strategy is able to allow a bigger reduction of the amount of consumed energy, the lack of QoS requirements causes a huge loss in terms of overall throughput achieved by users. On the other hand, the EE $Q o S$ solution can obtain an acceptable throughput performance, holding up to the Closest BS Association reference case, while guaranteeing fair energy savings. Moreover, the flexible management of user association and radio resources adopted by the EE $Q o S$ strategy allows to greatly exceed the QoS requirements in terms of target throughput. With an additional $1.5 \%$ energy expense, EE QoS solution is able to bring up to a $44.3 \%$ improvement for the throughput with respect to the $E E$ strategy, still guaranteeing up to $8 \%$ less energy consumption compared to the Closest BS Association.

\section{CONCLUSION}

In this paper an energy saving solution for LTE heterogeneous networks is presented and implemented in a multiobjective optimization framework. The energy saving is obtained by adapting the number of active macro and micro base stations to traffic variations. Since each cell needs the knowledge of traffic variations in its coverage area, a traffic forecast technique is used. The network is able to adapt itself to the capacity requested by users at different times of the day, solving the power consumption minimization problem with QoS target requirements and guaranteeing a minimum number of $\mathrm{BS}$ sleep mode operations for macro and micro cell subsets. The proposed EE $Q O S$ strategy affords performance very close to the optimum solution, particularly in terms of active base stations. By optimizing the network configuration in terms of BS sleep mode switchings, this study shows the positive impact of long term sleep solutions in a heterogeneous cellular network scenario, holding down the related signaling and handover traffic overhead as well as the negative impact on operations and maintenance activities, which involve operational performance, monitoring and control of site operations. Combination of different energy saving features and additional optimization objectives is considered as a future work.

\section{REFERENCES}

[1] L. Saker, S.-E. Elayoubi, and T. Chahed, "Minimizing energy consumption via sleep mode in green base station," in IEEE Wireless Communications and Networking Conference (WCNC), April 2010, pp. $1-6$.

[2] S. Morosi, P. Piunti, and E. Del Re, "Improving cellular network energy efficiency by joint management of sleep mode and transmission power," in 24th Tyrrhenian International Workshop on Digital Communications - Green ICT (TIWDC), Sept 2013, pp. 1-6.

[3] M. A. Marsan, L. Chiaraviglio, D. Ciullo, and M. Meo, "Multiple daily base station switch-offs in cellular networks," in Fourth International Conference on Communications and Electronics (ICCE), Aug 2012, pp. 245-250.

[4] J. Wu, Y. Zhang, M. Zukerman, and E. K. N. Yung, "Energy-efficient base-stations sleep-mode techniques in green cellular networks: A survey," IEEE Communications Surveys Tutorials, vol. 17, no. 2, pp. 803 826, Second quarter 2015.

[5] H. Tabassum, U. Siddique, E. Hossain, and M. J. Hossain, "Downlink performance of cellular systems with base station sleeping, user association, and scheduling," IEEE Transactions on Wireless Communications, vol. 13 , no. 10 , pp. 5752-5767, Oct 2014.

[6] E. Bjornson, E. Jorswieck, M. Debbah, and B. Ottersten, "Multiobjective signal processing optimization: The way to balance conflicting metrics in 5g systems," IEEE Signal Processing Magazine, vol. 31, no. 6, pp. 14-23, Nov 2014.

[7] P. Piunti, M. Dolfi, S. Morosi, S. Jayousi, and E. Del Re, "Performance evaluation of an energy efficient rrm strategy in heterogeneous cellular networks," in IEEE 25th International Symposium on Personal, Indoor and Mobile Radio Communications (PIMRC), Sept 2014, pp. 1-5.

[8] A. S. Alam and L. S. Dooley, "A scalable multimode base station switching model for green cellular networks," in Wireless Communications and Networking Conference (WCNC), 2015 IEEE, March 2015, pp. 878-883.

[9] S. Morosi, P. Piunti, and E. Del Re, "Sleep mode management in cellular networks: a traffic based technique enabling energy saving," Transactions on Emerging Telecommunications Technologies, vol. 24, no. 3, pp. 331-341, 2013. [Online]. Available: http://dx.doi.org/10.1002/ett.2621

[10] P. Piunti, C. Cavdar, S. Morosi, K. Teka, E. Del Re, and J. Zander, "Energy efficient adaptive cellular network configuration with qos guarantee," in IEEE International Conference on Communications (ICC), June 2015, pp. 68-73.

[11] “EARTH WP2 D2.3," Tech. Rep., May 2012.

[12] G. Auer, V. Giannini, C. Desset, I. Godor, P. Skillermark, M. Olsson, M. Imran, D. Sabella, M. Gonzalez, O. Blume, and A. Fehske, "How much energy is needed to run a wireless network?" IEEE Wireless Communications, vol. 18, no. 5, pp. 40-49, October 2011.

[13] J. Zander, "Performance of optimum transmitter power control in cellular radio systems," IEEE Transactions on Vehicular Technology, vol. 41, no. 1, pp. 57-62, Feb 1992.

[14] C. Bliek1ú, P. Bonami, and A. Lodi, "Solving mixed-integer quadratic programming problems with ibm-cplex: a progress report," 2014.

[15] "Further advancements for E-UTRA physical layer aspects v.9.0.0," 3GPP TR 36.814, 2010. 